\title{
Self-reported Wheat Sensitivity in Irritable Bowel Syndrome and Healthy Subjects: Prevalence of Celiac Markers and Response to Wheat-free Diet
}

\author{
Pavan Dhoble, ${ }^{1}$ Philip Abraham, ${ }^{1 *}$ Devendra Desai, ${ }^{1}$ Anand Joshi, ${ }^{1}$ Tarun Gupta, ${ }^{1}$ Shachish Doctor, ${ }^{1}$ Anand Deshpande, ${ }^{2}$ \\ and Rajeshwari Basavanna ${ }^{2}$ \\ ${ }^{I}$ Division of Gastroenterology and ${ }^{2}$ Department of Transfusion Medicine, P D Hinduja Hospital, Mumbai, India
}

\begin{abstract}
Background/Aims
Most patients with irritable bowel syndrome (IBS) report food-related aggravation of symptoms. Wheat/gluten is one of the most commonly incriminated. We studied the prevalence of self-reported wheat sensitivity in patients with IBS and in a healthy population from a region in India consuming mixed-cereal diets, correlated it with serological and human leukocyte antigen (HLA) markers of celiac disease, and evaluated the response to a wheat-free diet.

\section{Methods}

We surveyed 204 patients with IBS and 400 healthy persons for self-reported wheat sensitivity. Testing for IgA anti-tissue transglutaminase and HLA DQ2 or DQ8 was done in individuals who reported wheat sensitivity. Consenting persons with wheat sensitivity were put on wheat-free diet and monitored for symptom change.
\end{abstract}

\section{Results}

Twenty-three of 204 patients with IBS (11.3\%) and none of the healthy subjects self-reported wheat sensitivity. Of 23 patients, 14 (60.9\%) were positive for HLA DQ2 or DQ8 and none for anti-tissue transglutaminase antibody. After 6 weeks on wheat-free diet, all 19 participating patients reported clinical improvement; fewer patients had bloating, diarrhea, constipation, and easy fatigue.

\section{Conclusions}

Eleven percent of patients with IBS self-reported wheat sensitivity. None of them had positive celiac serology; $60.9 \%$ were positive for HLA DQ2 and DQ8, suggesting a possible genetic basis. All of them improved symptomatically on a wheat-free diet.

(J Neurogastroenterol Motil 2021;27:596-601)

Key Words

Food sensitivity; Gluten sensitivity; HLA typing; Non-celiac gluten sensitivity; Transglutaminases

Received: April 23, 2020 Revised: October 6, 2020 Accepted: November 24, 2020

(c) This is an Open Access article distributed under the terms of the Creative Commons Attribution Non-Commercial License (http://creativecommons. org/licenses/by-nc/4.0) which permits unrestricted non-commercial use, distribution, and reproduction in any medium, provided the original work is properly cited.

*Correspondence: Philip Abraham, MD

Division of Gastroenterology, P D Hinduja Hospital and MRC, V S Marg, Mahim, Mumbai 400016, India Tel: +91-22-24447104, Fax: +91-22-24440425, E-mail: dr_pabraham@hindujahospital.com 


\section{Introduction}

Exacerbation of symptoms by food intake, either as a direct or delayed reaction, has been reported by more than one-half of patients with irritable bowel syndrome (IBS). ${ }^{1}$ Most patients $(84.0 \%)$ report meal-related symptoms to at least 1 food item; these are associated with higher symptom severity scores and a reduced quality of life. ${ }^{2}$ A group of poorly absorbed short-chain carbohydrates including fructose and lactose, fructans, galacto-oligosaccharides, and polyols or sugar alcohols (fermentable oligosaccharides, disaccharides, monosaccharides, and polyols [FODMAPs]) has been commonly incriminated in causation of symptoms.

Gluten-based products are cited as an offending culprit by roughly 1 in 4 patients with IBS. $^{2}$ A study in Indian patients with IBS symptoms reported $0.8 \%$ prevalence of celiac disease (CD) and $5.2 \%$ prevalence of potential CD. ${ }^{3}$ Patients in this study were predominantly from the northern parts of the country where wheat is a staple cereal; the findings may not be reproducible elsewhere in India with mixed dietary habits (in southern India, for example, rice is the staple cereal). While a small fraction of patients with IBS (especially those with the diarrhea variety; IBS-D) may in fact have $\mathrm{CD}$, more patients reporting wheat sensitivity do not have the serological or histological features of CD (non-celiac gluten sensitivity [NCGS]). ${ }^{4}$ NCGS is increasingly reported in the West among IBS subjects; there are also reports from other countries of this phenomenon. ${ }^{3,5}$ Human leukocyte antigen (HLA) DQ2 and DQ8, considered as markers of $\mathrm{CD}$, increase the propensity to gluten intolerance even in NCGS.

The entity of NCGS or wheat sensitivity is gaining interest in the lay and medical literature. ${ }^{8,9}$ Efforts have been made during the past 5 years to define the diagnostic criteria for NCGS. ${ }^{40}$ Surveys in the West have shown that $5 \%$ to $15 \%$ of the general population consider themselves as gluten sensitive, and a fraction of them even go on a gluten-free diet (GFD) (may not be strictly) by themselves. ${ }^{4}$ Most of these individuals have had no formal testing for their believed sensitivity. ${ }^{4,11}$

NCGS is also recognized in patients with IBS, especially in those with IBS-D and the mixed (IBS-M) variety. ${ }^{9,12}$ Patients with IBS often place themselves on a wheat- or GFD of their own volition even in the absence of diagnosed CD. ${ }^{13}$ One of the consequences of such a trend is that it may lead to diagnostic delay, considerable waste of resources, and maybe nutritional deprivation.

The cosmopolitan city of Mumbai has a mixed population that consumes both rice- and wheat-based diets. In this population, we studied the prevalence of self-reported wheat sensitivity in patients with IBS and in healthy subjects; we also studied the prevalence of a sensitive serological test for CD (IgA anti-tissue transglutaminase $[\mathrm{tTg}])$ as well as of HLA DQ2 and DQ8. We then studied the response to a wheat-free diet (WFD) in IBS patients with selfreported wheat sensitivity. There has been no data on these aspects from such a population.

\section{Materials and Methods}

The clinical study was conducted in the Division of Gastroenterology, and the HLA studies at the Department of Transfusion Medicine. The protocol and related documents were approved by the institutional review board (Project No. 1026-16-PA).

All patients with IBS (Rome III criteria), aged 17 to 80 years, seen in the outpatient clinic between October 2016 and November 2019, were screened for enrollment in this prospective observational study.

\section{Primary and Secondary Outcomes}

Our primary outcome measure was to ascertain and compare the prevalence of self-reported wheat sensitivity in patients with IBS and in a healthy population. As secondary outcome measures, we compared the prevalence of self-reported wheat sensitivity in the subgroups of patients with IBS, namely, IBS-D, IBS-constipation (IBS-C), and IBS-M. In addition, we ascertained the prevalence of serological marker (IgA anti-t Tg) and HLA markers (DQ2 and DQ8) for CD in patients with IBS and self-reported wheat sensitivity, and the prevalence of HLA DQ2 and DQ8 in a healthy population. Patients with IBS and self-reported wheat sensitivity were then kept on a WFD to ascertain response to it.

\section{Study Design and Protocol}

With a reported average prevalence of NCGS of $5.0 \%$ in the general population and approximately $10.0 \%$ in IBS patients, ${ }^{14}$ a minimum required sample size of 185 IBS patients was calculated at $95 \%$ confidence level and $80 \%$ power, using ClinCalc Sample Size Calculator (www.clincalc.com). Considering $10 \%$ dropout, the final required sample size was 204 IBS patients.

Thus, 204 consecutive consenting patients with IBS were enrolled for a pro forma survey on self-reported wheat sensitivity. With enrolment ratio of 1:2, 400 healthy adult subjects (defined as "never consulted for chronic, ie, more than 6 months' duration, gastrointestinal complaint"), aged 17 to 80 years, also participated in the survey; they were identified by convenience sampling from 
the community. Consecutive consenting persons with self-reported wheat sensitivity were counseled for testing for serological and HLA markers of CD, and were also counseled for a 6-week trial with WFD. The 6-week duration was chosen because it has been shown that in $\mathrm{CD}$, most patients (77\%) resolve their clinical symptoms within a month after commencing a GFD. ${ }^{15}$

Patients with previously diagnosed CD and those with IBS on self-imposed gluten restriction in the diet were excluded from serological/HLA testing and from the trial on WFD.

Written informed consent was taken from all patients and healthy subjects participating in the one-point pro forma survey. Additional consent was taken from those who agreed to provide blood samples ( $8 \mathrm{~mL}$ ) for testing for IgA anti-tTg and HLA DQ2 and DQ8, as well as those who agreed to participate in the trial with WFD. Those willing to participate in the trial with WFD were referred to a dietician who provided information on how to undertake such a diet.

The IBS Severity Scoring System (IBS-SSS) ${ }^{15}$ was used to assess the severity of IBS symptoms; scores range from 0 to 500 with higher scores indicating more severe symptoms. For the purpose of analysis, IBS patients were divided into 3 groups according to the following cut-off values: $<175$ mild IBS; 175-300 moderate IBS; and $>300$ severe IBS. The wheat-sensitive IBS patients were given the IBS-SSS questionnaire to complete on Day 0 (day before commencing WFD), and then during the WFD period at 4 and 6 weeks. After 6 weeks, patients were advised to return the questionnaire. A reduction of 50 points on the IBS-SSS was considered clinical improvement.

Following these assessments patients in whom IBS symptoms had improved were asked whether they were planning to continue with a WFD for the foreseeable future (yes/no answer). In those who answered "yes," a re-consultation was done 3 months later to assess sustainability of the diet and symptom scores.

Table 1. Distribution of Subtypes of Irritable Bowel Syndrome as per Wheat Sensitivity

\begin{tabular}{cccc}
\hline $\begin{array}{c}\text { IBS } \\
\text { subtypes }\end{array}$ & $\begin{array}{c}\text { Total } \\
(\mathrm{N}=204)\end{array}$ & $\begin{array}{c}\text { Non-wheat- } \\
\text { sensitive patients } \\
(\mathrm{n}=181)\end{array}$ & $\begin{array}{c}\text { Wheat-sensitive } \\
\text { patients } \\
(\mathrm{n}=23)\end{array}$ \\
\hline IBS-D & $82(40)$ & $72(40)$ & $10(44)$ \\
IBS-C & $61(30)$ & $52(29)$ & $9(39)$ \\
IBS-M & $61(30)$ & $57(31)$ & $4(17)$ \\
\hline
\end{tabular}

IBS-D, - $\mathrm{C}$ and - $\mathrm{M}$ are subtypes diarrhea predominant-, constipation predominant-, and mixed irritable bowel syndrome (IBS).

Data are presented as $\mathrm{n}(\%)$.

No significant difference in distribution between the three IBS subtypes. $(P=0.300)$.

\section{Statistical Methods}

Quantitative parameters are expressed as mean (SD), and qualitative variables as numbers and frequencies or percentages. Student's $t$ test was used for quantitative variables, and chi-square test and Fisher's exact test for qualitative variables, as appropriate. A $P$-value of less than 0.05 was considered statistically significant.

\section{Results}

The 204 IBS patients in the pro forma survey included 119 men (58.3\%); the 400 healthy subjects in the survey included 276 men $(69.0 \%)$. There was no difference in proportions of males and females between the subtypes of IBS. The median age was 36 years (interquartile range $=17$ ) in IBS patients and 40 years (interquartile range $=16)$ in the healthy subjects.

\section{Wheat Sensitivity}

Twenty-three (11.3\%) of the 204 patients with IBS self-reported wheat sensitivity (Table 1); none of the 400 healthy subjects selfreported wheat sensitivity.

\section{Symptom Analysis}

Table 2 shows the difference in symptoms between non-wheatsensitive and wheat-sensitive patients with IBS. Only the difference in bloating and joint pain was statistically significant, being higher in the wheat-sensitive group. In most patients with IBS, bloating was the predominant symptom (76.0\%) including 132 of 181 (73.0\%) in the non-wheat-sensitive group and all $23(100.0 \%)$ in the wheatsensitive group. Bloating was more common $(P<0.001)$ in IBS-

Table 2. Symptoms as per Wheat Sensitivity

\begin{tabular}{lccc}
\hline \multicolumn{1}{c}{ Symptoms } & $\begin{array}{c}\text { Non-wheat- } \\
\text { sensitive patients } \\
(\mathrm{n}=181)\end{array}$ & $\begin{array}{c}\text { Wheat-sensitive } \\
\text { patients } \\
(\mathrm{n}=23)\end{array}$ & $P$-value \\
\hline Bloating & $132(73)$ & $23(100)$ & 0.001 \\
Diarrhea & $72(40)$ & $10(43)$ & 0.730 \\
Constipation & $52(29)$ & $9(39)$ & 0.300 \\
Mixed (constipation & $57(31)$ & $4(17)$ & 0.170 \\
$\quad$ & & & \\
and diarrhea) & $31(17)$ & $5(22)$ & 0.290 \\
Abdominal pain & $80(44)$ & $10(43)$ & 0.940 \\
Easy tiredness & $8(4)$ & $2(9)$ & 0.800 \\
Nausea & $34(19)$ & $6(3)$ & 0.690 \\
Headache & $26(14)$ & $7(30)$ & 0.048 \\
Joint pain & &
\end{tabular}

Data are presented as $\mathrm{n}(\%)$. 
Table 3. Symptom Distribution Before and at 6 Weeks on Wheat-free Diet in Wheat-sensitive Patients With Irritable Bowel Syndrome

\begin{tabular}{lccr}
\hline \multirow{2}{*}{ Symptoms } & \multicolumn{2}{c}{ Wheat-free diet } & P-value \\
\cline { 2 - 3 } & Before & At 6 wk & \\
\hline Bloating & 19 & 0 & $<0.001$ \\
Diarrhea & 8 & 2 & 0.027 \\
Constipation & 7 & 1 & 0.016 \\
Mixed & 4 & 1 & 0.140 \\
Abdominal pain & 5 & 2 & 0.200 \\
Easy tiredness & 10 & 4 & 0.043 \\
Nausea & 3 & 2 & 0.460 \\
Headache & 6 & 4 & 0.460 \\
Joint pains & 7 & 5 & 0.480 \\
\hline
\end{tabular}

Data are presented as number.

D patients (71 of $82[86.6 \%]$ ) as compared to IBS-C (44 of 61 [72.1\%]) and IBS-M (40 of 61 [65.6\%]).

\section{IgA Anti-tissue Transglutaminase Serology}

None of the 23 self-reported wheat-sensitive patients tested positive for IgA anti-t Tg.

\section{Human Leukocyte Antigen DQ2/DQ8}

Of 23 IBS patients who self-reported wheat-sensitivity, 13 patients ( 8 males), ie, $56.5 \%$, were positive for HLA DQ2 haplotype and 1 female (4\%) for HLA DQ8 haplotype. HLA DQ positivity was higher in IBS-D (10 of 10 patients) than in IBS-C (4 of 9 patients), but the difference was not significant $(P=0.057)$.

\section{Response to Wheat-free Diet}

Of 23 IBS patients who self-reported wheat sensitivity, 4 did not consent for the 6-week WFD trial. After 6 weeks of WFD in the 19 consenting patients, the number of patients with bloating, diarrhea, constipation, and easy tiredness was significantly decreased. Bloating was the most commonly reported (all 19 patients) improved symptom (Table 3).

\section{Irritable Bowel Syndrome Severity Scoring System Pre and Post Wheat-free Diet}

Twelve of 19 patients $(63.2 \%)$ had severe (> 300) IBS-SSS, $6(31.6 \%)$ had moderate (176-300) IBS-SSS, and only $1(5.3 \%)$ had mild (75-175) IBS-SSS before starting the WFD trial. Post 6 weeks of WFD, all 19 patients had clinical improvement (50-point reduction on IBS-SSS): only 1 patient $(5.3 \%)$ had severe IBS-SSS, $2(10.5 \%)$ had moderate IBS-SSS, and 16 patients (84.2\%) had mild IBS-SSS $(P<0.001)$. All 10 HLA DQ2-positive patients (8 IBS-D and 2 IBS-C) showed IBS-SSS improvement (9 with severe IBS-SSS and 1 with moderate IBS-SSS changed to mild IBSSSS) on WFD.

All 19 patients were contacted at 3 months after completion of the 6-week WFD trial. Thirteen of them were following a wheatrestricted diet as per tolerance; 6 with mild symptoms planned to continue WFD long term.

\section{Discussion}

Patients with IBS often attempt to identify foods that induce symptoms. There are no clear guidelines on what types of elimination diets should be prescribed empirically to them, and the types of food that should be included in a challenge. Elimination diets do not have mechanistic evidence or clinical evidence to defend their continued use $^{16}$ and may lead to nutritional inadequacy. In a Norwegian study, $12.0 \%$ of patients with IBS were considered to have a nutritionally inadequate diet due to exclusions. ${ }^{17}$ Interest in restrictive diets has focused on lactose, FODMAPs, and gluten; gluten has recently attracted the most attention. Many people, including celebrities, have endorsed gluten-free diets for the relief of IBS symptoms. $^{18}$

We surveyed the prevalence of self-reported wheat sensitivity among patients with IBS and a healthy cohort, and correlated that with serological and genetic markers of CD. Our study is the only one from India to evaluate the prevalence of self-reported wheat sensitivity in IBS patients and healthy subjects. Mumbai is a cosmopolitan city, with residents hailing from all over the country and consuming mixed diets; the population we studied was therefore likely to be representative of the country. We report $11.3 \%$ prevalence of self-reported wheat sensitivity among IBS patients, which is comparable to globally reported prevalence rates. ${ }^{2,18-21}$ None of the healthy individuals reported wheat sensitivity.

None of our IBS patients who self-reported wheat sensitivity was positive for anti-tTg antibody. This was in contrast to the reported prevalence of $6.1 \%$ among IBS patients in the study from Delhi. ${ }^{3}$

Fourteen $(60.9 \%)$ of the 23 wheat-sensitive IBS patients were positive for HLA DQ2 or DQ8, suggesting a possible susceptibility to wheat sensitivity-related disease. This prevalence was not significantly higher when compared to the $44.7 \%$ prevalence in healthy kidney donors whose data were available in our HLA Lab (Supplementary Material); a type II error is possible because of the small number of IBS patients. There is wide variation in reported 
HLA positivity in different parts of the world. ${ }^{20,22-25}$ As reported by Wahnschaffe et al, ${ }^{26}$ we too noted better response to WFD in those who were HLA-DQ2 haplotype positive.

The term non-celiac "gluten" sensitivity continues to be in common use ${ }^{27}$ but is a misnomer in those who report wheat sensitivity, as components of wheat other than gluten may be responsible for the symptoms. ${ }^{24,27}$ In their review on the content of FODMAPs in the Indian diet, Mehtab et $\mathrm{al}^{28}$ classified wheat as a high-FODMAPs source. Sharma et al ${ }^{29}$ reported that close to $14.4 \%$ of Indian patients with IBS (and 2.5\% of normal individuals) had intolerance to fructose. Hence our preference for the term "self-reported wheat sensitivity" in these individuals.

Our study has limitations: it was not a randomized controlled trial; a future study could prospectively compare the outcome in those who continued with wheat versus those who went on a wheatfree diet, among those self-reporting wheat sensitivity. Secondly, we do not have data on duodenal histology in the patients with IBS who self-reported wheat sensitivity. Finally, the data on HLA DQ2 and DQ8 in healthy individuals could not be obtained because they did not have wheat sensitivity, depending on the study design.

In conclusion, $11.3 \%$ of patients with IBS self-reported wheat sensitivity in our study; this figure is far in excess of the reported incidence of CD in patients with IBS in northern India. ${ }^{3}$ None of them had positive serologic marker for CD (NCGS), but 60.9\% (versus $44.7 \%$ in healthy kidney donors) had a predisposing genetic factor (HLA DQ2 or DQ8). Wheat-free diet (which avoids not only gluten but also non-gluten proteins and fructans) led to significant improvement in symptoms, in patients who self-reported wheat sensitivity, especially in those with HLA DQ2 haplotype. None of the surveyed 400 healthy subjects self-reported wheat sensitivity. Our study is probably the only study from India exploring self-reported wheat sensitivity and correlating symptoms with serological and HLA markers of CD; it was done in a region that has a mixed rice- and wheat-eating population.

We recommend that HLA testing alone should not be considered for screening for $\mathrm{CD}$. Studies are needed to evaluate intestinal mucosal injury in subjects with self-reported wheat sensitivity with or without HLA markers. While wheat-free diet can lead to significant improvement in symptoms in IBS patients who self-reported wheat sensitivity, health professionals should educate their persons who undertake gluten-free diet on their own regarding the uncertainty of their diagnosis; these subjects can titrate their exposure to gluten/wheat as tolerated and should be warned that inappropriate dietary restrictions can cause nutritional deficiencies. Follow-up studies are required in those with self-reported wheat sensitivity with or without HLA markers, to determine the likelihood of evolving into $\mathrm{CD}$.

\section{Supplementary Material}

Note: To access the supplementary material mentioned in this article, visit the online version of Journal of Neurogastroenterology and Motility at http://www.jnmjournal.org/, and at https://doi. org/10.5056/jnm20086.

Acknowledgements: Part of the data in this article was presented as an e-poster at the Asia-Pacific Digestive Week, Kolkata, India, 2019 (J Gastroenterol Hepatol 2019;34(suppl 3):836).

Financial support: The study was supported by an intramural grant from the National Health and Education Society (NHES). The NHES had no role in the conduct of the study, analysis of data, or manuscript writing.

\section{Conflicts of interest: None.}

Author contributions: Pavan Dhoble, Philip Abraham, Devendra Desai, Tarun Gupta, and Shachish Doctor: conception and design of the study; Pavan Dhoble, Philip Abraham, Devendra Desai, Anand Deshpande, and Rajeshwari Basavanna: analysis and interpretation of the data; Pavan Dhoble, Philip Abraham, and Devendra Desai: drafting the article or revising it critically for important intellectual content; and Pavan Dhoble, Philip Abraham, Devendra Desai, Anand Joshi, Tarun Gupta, Shachish Doctor, Anand Deshpande, and Rajeshwari Basavanna: final approval of the version to be published.

\section{References}

1. Zar S, Kumar D, Benson MJ. Food hypersensitivity and irritable bowel syndrome. Aliment Pharmacol Ther 2001;15:439-449.

2. Böhn L, Störsrud S, Törnblom H, Bengtsson U, Simrén M. Selfreported food-related gastrointestinal symptoms in IBS are common and associated with more severe symptoms and reduced quality of life. Am J Gastroenterol 2013;108:634-641.

3. Sharma H, Verma AK, Das P, Dattagupta S, Ahuja V, Makharia GK. Prevalence of celiac disease in Indian patients with irritable bowel syndrome and uninvestigated dyspepsia. J Dig Dis 2015;16:443-448.

4. Sapone A, Bai JC, Ciacci C, et al. Spectrum of gluten-related disorders: consensus on new nomenclature and classification. BMC Med 2012;10:13

5. Wang H, Zhou G, Luo L, et al. Serological screening for celiac disease in adult Chinese patients with diarrhea predominant irritable bowel syndrome. Medicine 2015;94:e1779. 
6. Aziz I, Trott N, Briggs R, North JR, Hadjivassiliou M, Sanders DS. Efficacy of a gluten-free diet in subjects with irritable bowel syndromediarrhea unaware of their HLA-DQ2/8 genotype. Clin Gastroenterol Hepatol 2016;14:696-703, e1.

7. Catassi C, Bai JC, Bonaz B, et al. Non-celiac gluten sensitivity: the new frontier of gluten related disorders. Nutrients 2013;5:3839-3853.

8. Makharia A, Catassi C, Makharia GK. The overlap between irritable bowel syndrome and non-celiac gluten sensitivity: a clinical dilemma. Nutrients 2015;7:10417-10426.

9. Verdu EF, Armstrong D, Murray JA. Between celiac disease and irritable bowel syndrome: the "no man's land" of gluten sensitivity. Am J Gastroenterol 2009; 104:1587-1594.

10. Catassi C, Elli L, Bonaz B, et al. Diagnosis of non-celiac gluten sensitivity (NCGS): the salerno experts' criteria. Nutrients 2015;7:4966-4977.

11. Volta U, Caio G, Tovoli F, De Giorgio R. Non-celiac gluten sensitivity: questions still to be answered despite increasing awareness. Cell Mol Immunol 2013;10:383-392.

12. Czaja-Bulsa G. Non coeliac gluten sensitivity: a new disease with gluten intolerance. Clin Nutr 2015;34:189-194.

13. Aziz I, Hadjivassiliou M, Sanders DS. The spectrum of noncoeliac gluten sensitivity. Nat Rev Gastroenterol Hepatol 2015;12:516-526.

14. Molina-Infante J, Santolaria S, Sanders DS, Fernández-Bañares F. Systematic review: noncoeliac gluten sensitivity. Aliment Pharmacol Ther 2015;41:807-820.

15. Francis CY, Morris J, Whorwell PJ. The irritable bowel severity scoring system: a simple method of monitoring irritable bowel syndrome and its progress. Aliment Pharmacol Ther. 1997;11:395-402.

16. McKenzie YA, Bowyer RK, Leach H, et al. British dietetic association systematic review and evidence-based practice guidelines for the dietary management of irritable bowel syndrome in adults (2016 update). J Hum Nutr Diet 2016;29:549-575.

17. Monsbakken KW, Vandvik PO, Farup PG. Perceived food intolerance in subjects with irritable bowel syndrome-- etiology, prevalence and consequences. Eur J Clin Nutr 2006;60:667-672.

18. Golley S, Corsini N, Topping D, Morell M, Mohr P. Motivations for avoiding wheat consumption in Australia: results from a population survey. Public Health Nutr 2015;18:490-499.
19. Aziz I, Lewis NR, Hadjivassiliou M, et al. A UK study assessing the population prevalence of self-reported gluten sensitivity and referral characteristics to secondary care. Eur J Gastroenterol Hepatol 2014;26:3339.

20. Volta U, Bardella MT, Calabrò A, Troncone R, Corazza GR. An Italian prospective multicenter survey on patients suspected of having non-celiac gluten sensitivity. BMC Med 2014;12:85.

21. Carroccio A, Giambalvo O, La Blasca F, Iacobucci R, D'Alcamo A, Mansueto P. Self-reported non-celiac wheat sensitivity in high school students: demographic and clinical characteristics. Nutrients 2017;9:771.

22. Massari S, Liso M, De Santis L, et al. Occurrence of nonceliac gluten sensitivity in patients with allergic disease. Int Arch Allergy Immunol 2011;155:389-394.

23. Brottveit M, Beitnes AC, Tollefsen S, et al. Mucosal cytokine response after short-term gluten challenge in celiac disease and non-celiac gluten sensitivity. Am J Gastroenterol 2013;108:842-850.

24. Biesiekierski JR, Peters SL, Newnham ED, Rosella O, Muir JG, Gibson PR. No effects of gluten in patients with self-reported non-celiac gluten sensitivity after dietary reduction of fermentable, poorly absorbed, short-chain carbohydrates. Gastroenterology 2013;145:320-328, e1-e3.

25. Kabbani TA, Vanga RR, Leffler DA, et al. Celiac disease or non-celiac gluten sensitivity? An approach to clinical differential diagnosis. Am J Gastroenterol 2014;109:741-746.

26. Wahnschaffe U, Schulzke JD, Zeitz M, Ullrich R. Predictors of clinical response to gluten-free diet in patients diagnosed with diarrhea-predominant irritable bowel syndrome. Clin Gastroenterol Hepatol 2007;5:844850.

27. Al-Toma A, Volta U, Auricchio R, et al. European society for the study of coeliac disease $(\mathrm{ESsCD})$ guideline for coeliac disease and other glutenrelated disorders. United European Gastroenterol J 2019;7:583-613.

28. Mehtab W, Agarwal A, Singh N, Malhotra A, Makharia GK. All that a physician should know about FODMAPs. Indian J Gastroenterol 2019;38:378-390.

29. Sharma A, Srivastava D, Verma A, Misra A, Ghoshal UC. Fructose malabsorption is not uncommon among patients with irritable bowel syndrome in India: a case-control study. Indian J Gastroenterol 2014;33:466470 . 